\title{
Respiratory Syncytial Virus Infection in High-risk Infants - an Update on Palivizumab Prophylaxis
}

\author{
Bernhard Resch* \\ Division of Neonatology, Department of Pediatrics, Medical University of Graz, Austria; Research Unit for Neonatal \\ Infectious Diseases and Epidemiology, Medical University of Graz, Austria
}

\begin{abstract}
Morbidity due to respiratory syncytial virus (RSV) disease is still high in infants and children worldwide during the first two to five years of life. Certain categories of high-risk infants with increased morbidity and mortality attributed to RSV disease have been identified and are included in national recommendations for prophylaxis with the monoclonal RSV antibody palivizumab. Most guidelines recommend palivizumab for preterm infants born less than or equal to 32 weeks gestational age with or without bronchopulmonary dysplasia, those born between 33 and 35 weeks gestational age with additional risk factors, and infants and children with hemodynamically significant congenital heart disease. Over the last years several rare diseases have been identified demonstrating high morbidity associated with RSV disease, thus, extension of guidelines for the prophylaxis with palivizumab for these patients with rare diseases including children with malignancy, congenital and acquired immune deficiency, Down syndrome, neuromuscular impairment, cystic fibrosis, congenital diaphragmatic hernia and other severe respiratory disease is increasingly discussed.
\end{abstract}

Efficacy of palivizumab prophylaxis is documented by meta-analysis, and different economic analyses demonstrate costeffectiveness of palivizumab for the most common indications during the first RSV season.

Keywords: Palivizumab, prophylaxis, respiratory syncytial virus, respiratory tract infection.

\section{INTRODUCTION}

Morbidity due to respiratory syncytial virus (RSV) disease is still high in infants and children worldwide during the first two to five years of life. RSV is the most common cause of bronchiolitis in infants and young children, and up to $80 \%$ of cases are attributable to RSV infection. In a recent meta-analysis the authors suggested that RSV caused up to 33.8 million acute lower respiratory tract infections (LRTI) among children under the age of five years each year, and this represents approximately $22 \%$ of all LRTI. At least 3.4 million cases of RSV related LRTI required hospitalization, and mortality was estimated to range between 66,000 and 199,000; with the majority (99\%) occurring in developing countries [1]. To define the imposition of hospitalizations related to RSV and non-RSV bronchiolitis in a tertiary-care children's hospital Garcia et al. [2] analyzed medical records of children younger than two years of age and hospitalized for bronchiolitis from 2002 to 2007 and identified the risk factors associated with severedisease by comparison of RSV with non-RSV associated bronchiolitis. Besides an increase of RSV associated bronchiolitis over the years RSV LRTI was associated with younger age, longer hospitalization, and

\footnotetext{
*Address correspondence to this author at the Division of Neonatology, Department of Pediatrics and Adolescent Medicine, Medical University of Graz, Auenbruggerplatz 34/2, A-8036 Graz, Austria; Tel: 0043316385 81134; Fax: 0043316385 12678;

E-mail: bernhard.resch@medunigraz.at
}

more often requirement of supplemental oxygen, intensive care, and mechanical ventilation. RSV and a history of prematurity were found to beindependent risk factors for severe disease [2]. The variability in disease severity is wide in children with bronchiolitis and prematurity, congenital heart diseases, chronic lung disease, and immune deficiencies are well known risk factors for severe disease. Nevertheless, the majority of hospitalized infants and even of those admitted to an intensive care unit are healthy term born infants. Recently, the influence of multiple viruses detected by real-time polymerase chain reaction was investigated in children less than 2 years of age with clinical signs and symptoms comparable to bronchiolitis over three cold seasons [3]. In total, out of 142 samples RSV was the virus most commonly detected $(73 \%)$ followed by rhinovirus found in 30 percent. More than one virus was detected in $41 \%$ of the samples and again $41 \%$ of those were dual infections with RSV and RV. Disease severity categorized as mild without the need for supportive treatment, moderate with need for supplemental oxygen and/or nasogastric feeding and severe with need for mechanical ventilation was not attributed to multiple viruses infection, thus, other factors like younger age might contribute to a larger extend to disease severity.

Palivizumab is a humanized monoclonal antibody that binds to the F-glycoprotein of the virus which is to $95 \%$ identical between RSV type A and B. Given monthly intramuscularly at a dosage of $15 \mathrm{mg} / \mathrm{kg}$ body weight over the RSV season (starting between October and November 
and ending between March and April and, thus, providing a total of 5 injections to cover the most significant seasonal activity of the virus) palivizumab significantly reduces severe RSV LRTI demonstrated by reduced RSV associated hospitalizations. According to most national guidelines palivizumab is routinely recommended for preterm infants with and without bronchopulmonary dysplasia and infants and children with hemodynamically significant congenital heart disease. Due to high costs associated with paliviumab prophylaxis in high-risk populations several limitations have been introduced by national boards despite the fact that palivizumab is the only tool against severe RSV disease and some economic studies have proven cost-effectiveness in certain high-risk populations $[4,5]$.

This review elucidates the latest evidence for palivizumab prophylaxis in high-risk infants and children published during the last years.

\section{PREMATURITY}

Preterm infants are known to be at increased risk for severe RSV LRTI necessitating hospitalization compared to term infants. This is in fact due to anatomic factors including smaller lung volumes, a reduced lung surface area, smaller airways and an increased thickness of air space walls on the one hand, and due to immunological factors including lower antibody titres by incomplete transfer of maternal antibodies via the placenta and a reduced cellular immunity resulting in a reduced viral clearance. In preterm infants without bronchopulmonary dysplasia a mean RSV related hospitalization rate of $9.3 \%$ from an analysis of 18 studies including more than 20,000 infants has been reported [6]. An analysis of the Canadian palivizumab registry reported on patients having siblings, those attending daycare, and those having either smokers or equal or more than five people in their household being at higher risk of an RSV-positive hospitalization. By use of hazard ratios the risk increased concurrently by a gradual increase in the number of risk factors [7]. Interestingly, comparing human metapneumovirus virus and RSV infection, young age below six months and crowded living were both associated with human metapneumovirus disease related hospitalization. Among hospitalized patients risk factors associated with severe human metapneumovirus disease included female sex, a history of prematurity, and genotype B infection. Risk factors for RSV hospitalization included age below six months, any comorbidity, and household crowding; breast-feeding and viral coinfection were found to be protective for RSV hospitalization [8]. Thus, some similarities were found between both infections regarding additional risk factors. Young age below six months and a history of prematurity were both associated with severe RSV disease among hospitalized children.

Palivizumab effectively reduces RSV related hospitalization rates in preterm infants. Among 13,310 patients included in the Canadian registry the overall rehospitalization rate was calculated with $1.55 \%$, and for preterm infants $1.36 \%$ [5]. Compliance with RSV prophylaxis varies; recently a high rate of $92.6 \%$ has been reported among preterm infants within the Canadian Registry of Palivizumab [9].
The American Academy of Pediatrics [10] recommends prophylaxis with palivizumabin case of preterm infants born equal or younger than 28 weeks of gestational age during the RSV season up to 12 months of chronological age and in case of preterm infants born between 29 and 31 weeks of gestational age up to 6 months of chronological age. Additionally the policy statement emphasizes that once an infant qualifies for palivizumabprophylaxis administration should be continued throughout the RSV season. In this case palivizumab should not be stopped by reaching either 6 or 12 months of age. Up to five monthly injections are recommended for infants in this age group. Between 32 and 35 weeks of gestational age prophylaxis might be considered for these infants in case of a chronological age of less than three months before the onset or duringthe RSV season. Additionally one of the two risk factors including day care attendance or siblings or other children younger than five years living in the same household should be evident. Infants in this gestational age group should be given palivizumab up to the age of 3 months and receive a maximum of three injections.

A recent article reported on a significant variability between patients regarding trough serum concentrations of palivizumab in clinical trials [11]. Partial season dosing was linked with an increased risk of RSV related hospitalization. For late-preterm infants, data suggested that the risk of RSV related hospitalizations remained elevated through at least 6 months of age. The authors concluded that late-preterm infants would benefit from palivizumab prophylaxis throughout the RSV season [11].

In order to determine the probability of RSV related hospitalization in preterm infants of 33 to 35 weeks of gestational age a prediction rule was developed using four predictors: family atopy (OR $1.9 ; 95 \% \mathrm{CI}, 1.1-3.2)$, birth period (OR 2.6; 1.6-4.2), breastfeeding (OR 1.7; 1.0-2.7) and siblings or daycare attendance (OR 4.7; 1.7-13.1). This was found by collecting data from two prospective birth cohorts from 41 hospitals in the Netherlands including 2421 infants [12]. Several risk scores and tools have been developed showing diverging results in the prediction of RSV related rehospitalization risk for predominantly late preterm infants, thus, influencing some national guidelines for palivizumab prophylaxis but not strictly being the basis for introduction in clinical routine [13-15]. In an US sample of infants of 32 to 35 weeks of gestational age during the 2007 to 2008 RSV season the prevalence of risk factors was estimated under various potential risk factors coverage policies from pharmacy providers [16]. Adding passive smoke exposure, crowded living conditions, and young chronological age to the list of risk factors (two or more of eight risk factors) increased the percentage of infants potentially receiving palivizumab prophylaxis up to $55.6 \%$ by increasing payer costs by only nine cents per-member-per-month. These criteria would identify more infants of 32 to 35 weeks of gestational age for palivizumab prophylaxis and the heightened costs might be acceptable, the authors concluded [16]. A recent systematic literature review identified environmental tobacco smoke exposure being a significant risk factor associated with RSV hospitalization especially in preterm infants [17]. Overall, out of 30 articles identified, the authors found significant evidence that environmental 
tobacco smoke exposure led to an increased risk of RSV related hospitalization and to an increased severity of illness among hospitalized infants and young children.

A meta-analysis on the association of RSV and pulmonary sequelae confirmed an association between RSV related hospitalization in young infantsand asthma and/or recurrent wheezing during the following years [18]. Fifteen studies including 82,008 infants of whom 1533 had documented RSV related hospitalization were selected for meta-analysis. Children with RSV disease at young age had a significantly higher incidence of asthma and or recurrent wheezing with an odds ratio of 3.84 (95\% CI 3.23-4.58). This association tended to decrease by increasing age of the children. In otherwise healthy preterm infants of 33 to 35 weeks gestational age results of a double blind placebo controlled trial including 429 infants revealed that palivizumab prophylaxis resulted in a significant reduction in wheezing days during the first year of life [19]. This finding either implicated RSV being an important trigger of recurrent wheezing or palivizumab being highly effective in reducing RSV associated wheezing both for the first 12 months of age.

\section{BRONCHOPULMONARY DYSPLASIA}

In preterm infants with bronchopulmonary dysplasia (BPD) a mean RSV related hospitalization rate of $19.8 \%$ from 8 studies including 2721 infants has been reported [6]. Additionally, the mean RSV LRTI associated mortality rate was remarkable being $1.5 \%$ (range 0 to $8.0 \%$ ). Palivizumab demonstrated a significant reduction of RSV related hospitalization rates down to $1.64 \%$ [7]. Most recommendations for preterm infants with BPD adhere to the policy statement of the AAP [10] that suggests palivizumab prophylaxis for infants and children younger than 24 months with BPD who necessitated medical therapy for their chronic lung disease including supplemental oxygen, bronchodilator, diuretic or chronic corticosteroid treatment within six months before the onset of the RSV season.

\section{CONGENITAL HEART DISEASE}

In infants and children with congenital heart disease (CHD) mean RSV rehospitalization rates of $7.8 \%$ with a mean RSV LRTI associated mortality rate of as high as $7.3 \%$ have been summarized recently from 11 studies including almost 13,000 children [20].

Children younger than 24 months of age having hemodynamically significant either cyanotic or acyanotic CHD represent candidates forprophylaxis with palivizumab. This is especially the case when they receive medication to control congestive heart failure or have moderate-to-severe pulmonary hypertension or cyanotic heart disease [8]. These recommendations were adopted from most national guidelines with differences mainly concerning the age of either 12 or 24 months [20].

\section{CHILDREN WITH CANCER}

Retrospectively over a 12-years period (1998 to 2009) 59 children with cancer were identified having had RSV infection at a median age of 5 years [21]. Within one month before RSV infection $53 \%$ of children had a hematologic malignancy, $32 \%$ had received hematopoietic stem cell transplantation, 39\% corticosteroids, and $76 \%$ cytotoxic chemotherapy. Additional risk factors included male gender and lymphocytopenia. Treatment with aerosolized ribavirin plus palivizumab was superior to aerosolized ribavirin alone in children with LRTI resulting in significantly better outcomes including reduced mortality rates.

\section{CYSTIC FIBROSIS}

Children with cystic fibrosis were reported to have more likely hospitalization and/or experience of deteriorating lung function during RSV season compared to children without cystic fibrosis [22]. RSV infection in patients with cystic fibrosis might lead to prolonged hospitalization with need for mechanical ventilation, evidence of persistent hypoxemia, and deterioration of lung function for several months following RSV associated LRTI. A Cochrane review identified only one randomized placebo controlled study that enrolled 186 infants and children younger than two years old and compared five monthly palivizumab injections (92 cases) to placebo (94 controls) over one RSV season [22]. At a follow-up of six months one infant in each group experienced RSV related hospitalization. At a follow-up of 12 months no differences in number of Pseudomonas bacterial colonization were observed. A more recent metaanalysis focused on morbidity due to RSV bronchiolitis and on the safety and efficacy of palivizumabin cystic fibrosis patients [23]. Of 354 patients having diagnosis of cystic fibrosis and palivizumab prophylaxis the hospital admission rate was found to be $1.8 \%$ (95\% CI $0.77-4.8 \%)$. The corresponding number in the non-treated groups was 463 patients with an admission rate of $12.6 \%$ (95\% CI 8.6$18.2 \% ; \mathrm{p}<0.001)$. Thus, palivizumab proved to be effective in the prevention of LRTI in children with diagnosis of cystic fibrosis.

\section{DOWN SYNDROME}

Down syndrome is a known risk factor for RSV hospitalization independent of its associated CHD [24, 25]. The CARESS registry identified 600 out of 13,300 children (having received at least one injection of palivizumab during the 2006 to $2012 \mathrm{RSV}$ seasons in Canada) as having diagnosis of Down syndrome [26]. Compared to other indications of palivizumab prophylaxis RSV hospitalization rates were low in Down syndrome children below 2 years of age following prophylaxis $(1.53 \%$ compared to $2.27 \%$ ) and hazard ratios were similar to those found in other indications. Interestingly, the proportion of Down syndrome children having received palivizumab in this registry was found to have increased almost 45 -fold (from $0.1 \%$ to $4.5 \%$ ).

\section{NEUROMUSCULAR IMPAIRMENT AND IMMUNE DEFICIENCY SYNDROMES}

There is a growing body of evidence that neuromuscular impairment is associated with severe RSV disease [27]. Factors that predispose to a more severe course of RSV disease include disturbances in the clearance of secretions from the airways mainly due to ineffective cough, weakness of respiratory muscles, and a high prevalence of gastro- 
oesophageal reflux and swallowing dysfunction leading to recurrent aspirations [28]. T-cell immunodeficiency commonly presents with severe pulmonary disease and infants with severe congenital or acquired immune deficiency syndromes have been demonstratedto shed viruses for a prolonged time period. RSV related LRTI have been reported to result in increased morbidity and mortality [26]. RSV hospitalization rates have been observed to occur 3 to 10 times more often in these high risk populations. Thus, prophylaxis with palivizumab during the first and/or the second RSV season might be indicated [29].

Recently, a cohort study (2006 to 2011) including RSVpositive immunocompromised pediatric inpatients reported on a mortality rate of $4.3 \%$ [28]. The patients who died (four infants and one child aged 12 years) were all diagnosed with community acquired RSV LRTI at admission. RSV was identified from tracheal samples or from postmortem lung biopsies. The diagnoses among these patients included severe combined immunodeficiency, uncharacterized immunodeficiency, chronic granulomatous disease, and solid organ transplantation, respectively.

\section{CONGENITAL DIAPHRAGMATIC HERNIA AND OTHER SEVERE RESPIRATORY DISEASES}

Thirty to fifty percent of infants with congenital diaphragmatic hernia survive in spite of pulmonary hypoplasia, pulmonary hypertension and extracorporeal membrane oxygenation with sequelae including chronic lung disease, childhood asthma and reduced lung function and exercise tolerance at a mean age of 12 years [30]. During their first year of life approximately a third of the infants wereprone to recurrent respiratory tract infections. RSV infection poses amajor threat to theseinfants because of residual, obstructive airway disease, thus, suggestingroutine prophylaxis for all these children aged <2 years [31]. Additionally, increased respiratory work-load, raised thoracic pressure when mechanical ventilation is needed, and an active inflammatory response during the course of severe RSV infection might further weaken either an intact diaphragm or a previous operative site and, thus, be a reason for the recurrence of diaphragmatic hernia after primary repair [31].

Recently, the off-label use of palivizumab for some pediatric patients with severe respiratory diseases was discussed by an expert group of pediatric pulmonologists by means of a two-round modified Delphi technique [32]. Consensus was reached in 56\% of the statements regarding appropriateness of palivizumab use in children with the following disorders: severe respiratory involvement due to neuromuscular disease, congenital or acquired immuno deficiency, storage disease, cystic fibrosis, diseases involving impaired ciliary clearance, esophageal atresia and/or tracheoesophageal fistula with the need for surgery, diaphragmatic hernia, bronchopulmonary malformations, severe tracheomalacia, lung transplantation (either recipients or patients in the waiting list for lung transplant), oxygendependency for severe interstitial lung disease and severe pulmonary hypertension.

\section{EFFICACY AND ECONOMIC ASPECTS OF PALIVIZUMAB}

Palivizumab was recently evaluated by a Cochrane metaanalysis [33] regarding effectiveness and safety of palivizumab prophylaxis and reduction of hospitalization due to RSV infection in high-risk infants and children. Additionally assessment of cost-effectiveness (or cost-utility) of palivizumab prophylaxis compared with no prophylaxis in infants and children of different groups at risk for severe RSV disease was done. Seven randomized controlled trials were available, three compared palivizumab with placebo including 2831 patients, and four compared palivizumab with motavizumab including 8265 patients. Palivizumab prophylaxis reduced significantly the rate of RSV hospitalizations (RR $0.49,95 \%$ CI 0.37 to 0.64 ); and when compared to motavizumab, palivizumab resulted in a nonsignificant increased risk of RSV hospitalizations (RR 1.36, 95\% CI 0.97 to 1.90 ). For economic evidence 34 studies were found to report cost-effectiveness and/or cost-utility data for palivizumab prophylaxis. There were considerable variations in modelling approaches were considerable across the studies resulting in large differences regarding costeffectiveness results. Thus, the authors concluded that costeffectiveness of palivizumab prophylaxis was highly depending on the costs used in the models and on the different cost-effectiveness threshold of the countries.

Another systematic review of the literature and economic evaluation was carried out recently trying to calculate the cost-effectiveness of palivizumab prophylaxis in children with or without BPD or CHD [34]. Analysis of 16,128 subgroups revealed cost-effectiveness at a threshold of $£ 30,000(€ \approx 36,500$ or $\$ \approx 50,000)$ per quality-adjusted lifeyears. On the one side the authors found that cost-effective subgroups for children without BPD or CHD had to additionally have at least two other risk factors different from gestational and birth age; and on the other side costeffectiveness was found for children with BPD or CHD without having any other risk factor. Prophylaxis with palivizumab was not money saving when used unselectively (mainly regarding the factor chronological age up to 2 years) either in children without or with BPD or CHD.

Examining four groups of premature infants without BPD or CHD [(1) less than 32 weeks of gestational age and up to six months of chronologic age; (2) 32 to 34 weeks and up to three months of chronological age, with use of the 2009 American Academy of Pediatrics risk factors; (3) 32 to 35 weeks and up to six months of age, with use of the 2009 American Academy of Pediatrics risk factors; and (4) 32 to 35 weeks and up to six months of age with equal or less than one risk factor] for cost utility regarding palivizumab prophylaxis revealed cost-effectiveness for publically and commercially insured, guideline-eligible, high-risk premature infants from a US national policy perspective [35]. Another cost-utility analysis of palivizumab in a Medicaid population showed palivizumab being either costsaving or cost-effective among current guideline-eligible infants in the Medicaid population when dosed consistent with the FDA-approved labeling, and did not demonstrate cost-effectiveness in infants of 32 to 35 weeks gestational 
BPD bronchopulmonary dysplasia; CHD congenital heart disease, $\mathrm{CF}$ cystic fibrosis

Impact trial, Pediatrics 1998; CHD trial, Feltes et al., J Pediatrics 2003; * Frogel et al., J Perinatol 2008; ** Cohen et al., PediatrCardiol 2008

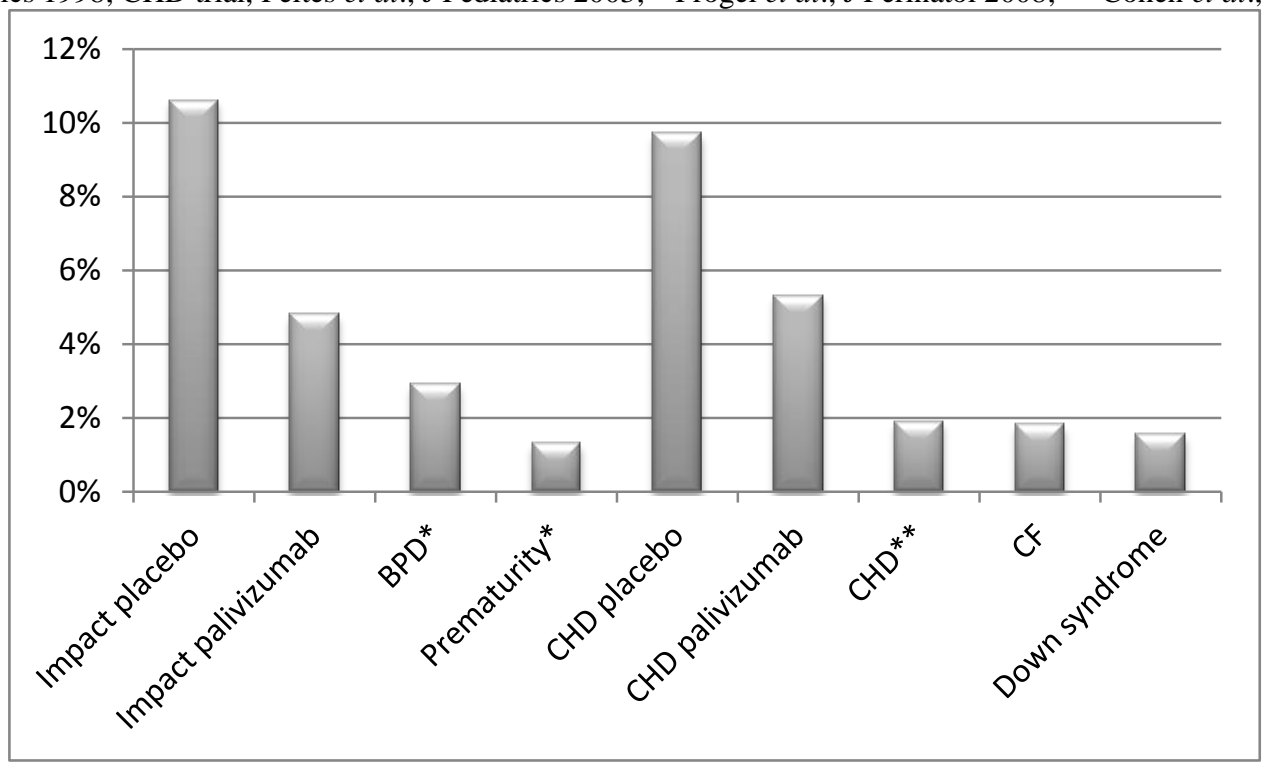

Fig. (1). Rates of RSV related hospitalizations by monthly prophylaxis with palivizumab under certain conditions.

age having equal or less than one risk factor [36]. A European cost-effectiveness study based on nationwide longterm epidemiologic data (including 1579 children hospitalized because of RSV LRTI during 16 seasons) from Austria confirmed these results for preterm infants below 33 weeks, those of 33 to 35 weeks of gestational age, those with $\mathrm{BPD}$, and at least those with $\mathrm{CHD}$; the latter demonstrating the lowest costs per quality-adjusted life years [37].

The reduction of RSV related hospitalization rates by monthly prophylaxis with palivizumab in certain conditions is shown in Fig. (1) [38-41].

\section{CONCLUSION}

There is a growing body of evidence on the use of palivizumab in special populations beyond the well-known recommendations for high-risk infants and children with a history of prematurity, BPD or CHD. In the light of the continuing burden of RSV disease, the limited symptomatic treatment modalities, and the long-lasting and still continuing research for a vaccine that still is not sight, palivizumab remains the only prophylactic measure to significantly reduce RSV hospitalization rates in these highrisk infants. Additionally palivizumab demonstrates the ability to ameliorate subsequent morbidity by reduction of post-RSV wheezing disorders. Nevertheless, further prospective studies in the group of infants and young children with these rare and uncommon medical illnesses are warranted to determine morbidity and mortality associated with RSV infection and further cost-effectiveness studies in these populations are needed.

\section{CONFLICT OF INTEREST}

I have received honoraria from Abbott Laboratories Limited for presentations on RSV infection and prophylaxis.

\section{ACKNOWLEDGEMENTS}

Declared none.

\section{REFERENCES}

[1] Nair H, Nokes DJ, Gessner BD, et al. Global burden of acute lower respiratory infections due to respiratory syncytial virus in young children: a systematic review and meta-analysis. Lancet 2010; 375: 1545-55.

[2] Garcia CG, Bhore R, Soriano-Fallas A, et al. Risk factors in children hospitalized with RSV bronchiolitis versus non-RSV bronchiolitis. Pediatrics 2010; 126: e1453-60.

[3] Brand HK, de Groot R, Galama JM, et al. Infection with multiple viruses is not associated with increased disease severity in children with bronchiolitis. Pediatr Pulmonol 2012; 47: 393-400.

[4] Resch B. Palivizumab for the prophylaxis of respiratory syncytial virus infection. Paed Health 2008; 2: 265-78.

[5] Resch B. Palivizumab in preventing respiratory syncytial virusrelated hospitalization in high-risk infants. Expert Rev Pharmacoecon Outcomes Res 2008; 8: 529-38.

[6] Resch B, Resch E, Müller W. Should respiratory care in preterm infants include prophylaxis against respiratory syncytial virus infection? The case in favour. Paediatr Respir Rev 2013; 14: 130-6.

[7] Paes B, Mitchell I, Li A, Harimoto T, Lanctôt KL. Respiratoryrelated hospitalizations following prophylaxis in the Canadian registry for palivizumab (2005-2012) compared to other international registries. Clin Dev Immunol 2013; 2013: 917068.

[8] Papenburg J, Hamelin MÈ, Ouhoummane N, et al. Comparison of risk factors for human metapneumovirus and respiratory syncytial virus disease severity in young children. J Infect Dis 2012; 206: 178-89.

[9] Paes B, Mitchell I, Li A, Lanctôt KL. CARESS Investigators. A comparative study of respiratory syncytial virus (RSV) prophylaxis in premature infants within the Canadian Registry of Palivizumab (CARESS). Eur J Clin Microbiol Infect Dis 2012; 31: 2703-11. 
[10] Committee on Infectious Diseases. From the American Academy of Pediatrics: Policy statements--Modified recommendations for use of palivizumab for prevention of respiratory syncytial virus infections. Pediatrics 2009; 124:1694-701.

[11] Makari D, Checchia PA, Devincenzo J. Rationale for full-season dosing for passive antibody prophylaxis of respiratory syncytial virus. Hum Vaccin Immunother 2013; 10. [Epub ahead of print].

[12] Blanken MO, Koffijberg H, Nibbelke EE, Rovers MM, Bont L. Dutch RSV Neonatal Network. Prospective validation of a prognostic model for respiratory syncytial virus bronchiolitis in late preterm infants: a multicenter birth cohort study. PLoS One 2013; 8: e59161.

[13] Doering G, Gusenleitner W, Belohradsky BH, Burdach S, Resch B, Liese JG. The risk of respiratory syncytial virus-related hospitalizations in preterm infants of 29 to 35 weeks' gestational age. Pediatr Infect Dis J 2006; 25: 1188-90.

[14] Simões EA, Carbonell-Estrany X, Fullarton JR, et al. European RSV Risk Factor Study Group. A predictive model for respiratory syncytial virus (RSV) hospitalisation of premature infants born at 33-35 weeks of gestational age, based on data from the Spanish FLIP Study. Respir Res 2008; 9: 78.

[15] Paes B, Steele S, Janes M, Pinelli J. Risk-scoring tool for respiratory syncytial virus prophylaxis in premature infants born at 33-35 completed weeks' gestational age in Canada. Curr Med Res Opin 2009; 25: 1585-91.

[16] Krilov LR, Palazzi DL, Fernandes AW, Klein RW, Mahadevia PJ. Prevalence of respiratory syncytial virus (RSV) risk factors and cost implications of immunoprophylaxis to infants 32 to 35 weeks gestation for health plans in the United States. Value Health 2010; 13: 77-86.

[17] DiFranza JR, Masaquel A, Barrett AM, Colosia AD, Mahadevia PJ. Systematic literature review assessing tobacco smoke exposure as a risk factor for serious respiratory syncytial virus disease among infants and young children. BMC Pediatr 2012; 12: 81.

[18] Régnier SA, Huels J. Association between respiratory syncytial virus hospitalizations in infants and respiratory sequelae: systematic review and meta-analysis. Pediatr Infect Dis J 2013; 32: 820-6.

[19] Blanken MO, Rovers MM, Molenaar JM, et al. Dutch RSV Neonatal Network. Respiratory syncytial virus and recurrent wheeze in healthy preterm infants. N Engl J Med 2013; 368: 17919 .

[20] Resch B, Michel-Behnke I. Respiratory syncytial virus infections in infants and children with congenital heart disease: update on the evidence of prevention with palivizumab. Curr Opin Cardiol 2013; 28: 85-91.

[21] Chemaly RF, Ghantoji SS, Shah DP, et al. Respiratory syncytial virus infections in children with cancer. J Pediatr Hematol Oncol 2013; Dec 10. [Epub ahead of print].

[22] Robinson KA, Odelola OA, Saldanha IJ, McKoy NA. Palivizumab for prophylaxis against respiratory syncytial virus infection in children with cystic fibrosis. Cochrane Database Syst Rev 2013 Jun 5; 6:CD007743.

[23] Sánchez-Solis M, Gartner S, Bosch-Gimenez V, Garcia-Marcos L. Is palivizumab effective as a prophylaxis of respiratory syncytial virus infections in cystic fibrosis patients? A meta-analysis. Allergol Immunopathol (Madr) 2013; Nov 11. [Epub ahead of print].

[24] Bloemers BL, van Furth AM, Weijerman ME, et al. Down syndrome: a novel risk factor for respiratory syncytial virus bronchiolitis--a prospective birth-cohort study. Pediatrics 2007; 120: e1076-81.
[25] Bloemers BL, Broers CJ, Bont L, Weijerman ME, Gemke RJ, van Furth AM. Increased risk of respiratory tract infections in children with Down syndrome: the consequence of an altered immune system. Microbes Infect 2010; 12: 799-808.

[26] Paes B, Mitchell I, Yi H, Li A, Lanctôt KL. CARESS Investigators. Hospitalization for respiratory syncytial virus illness in Down syndrome following prophylaxis with palivizumab. Pediatr Infect Dis J 2014; 33: e29-33.

[27] Simon A, Prusseit J, Müller A. Respiratory syncytial virus infection in children with neuromuscular impairment. Open Microbiol J 2011; 5: 155-8.

[28] Resch B, Manzoni P, Lanari M. Severe respiratory syncytial virus (RSV) infection in infants with neuromuscular diseases and immune deficiency syndromes. Paediatr Respir Rev 2009; 10: 14853.

[29] Fauroux B. Special populations. Paediatr Respir Rev 2009; 10 (Suppl 1): 21-2.

[30] Asner S, Stephens D, Pedulla P, Richardson SE, Robinson J, Allen U. Risk factors and outcomes for respiratory syncytial virus-related infections in immunocompromised children. Pediatr Infect Dis J 2013; 32: 1073-6.

[31] Manzoni P, Paes B, Resch B, Carbonell-Estrany X, Bont L. High risk for RSV bronchiolitis in late preterms and selected infants affected by rare disorders: a dilemma of specific prevention. Early Hum Dev 2012; 88 (Suppl 2): S34-41.

[32] Gaboli M, de la Cruz OA, de Agüero MI, Moreno-Galdó A, Pérez GP, de Querol MS. Use of palivizumab in infants and young children with severe respiratory disease: a delphi study. Pediatr Pulmonol 2013 Jun 18. doi: 10.1002/ppul.22826 [Epub ahead of print].

[33] Andabaka T, Nickerson JW, Rojas-Reyes MX, Rueda JD, BacicVrca V, Barsic B. Monoclonal antibody for reducing the risk of respiratory syncytial virus infection in children. Cochrane Database Syst Rev 2013; 4: CD006602.

[34] Wang D, Bayliss S, Meads C. Palivizumab for immunoprophylaxis of respiratory syncytial virus (RSV) bronchiolitis in high-risk infants and young children: a systematic review and additional economic modelling of subgroup analyses. Health Technol Assess. 2011; 15: iii-iv, 1-124.

[35] Mahadevia PJ, Masaquel AS, Polak MJ, Weiner LB. Cost utility of palivizumab prophylaxis among pre-term infants in the United States: a national policy perspective. J Med Econ 2012; 15: 987-96.

[36] Weiner LB, Masaquel AS, Polak MJ, Mahadevia PJ. Costeffectiveness analysis of palivizumab among pre-term infant populations covered by Medicaid in the United States. J Med Econ 2012; 15: 997-1018.

[37] Resch B, Sommer C, Nuijten MJ, et al. Cost-effectiveness of palivizumab for respiratory syncytial virus infection in high-risk children, based on long-term epidemiologic data from Austria. Pediatr Infect Dis J 2012; 31: e1-8.

[38] The Impact-RSV Study Group. Palivizumab, a humanized respiratory syncytial virus monoclonal antibody, reduces hospitalization from respiratory syncytial virus infection in highrisk infants. Pediatrics 1998; 102: 531-7.

[39] Frogel M, Nerwen C, Cohen A, VanVeldhuisen P, Harrington M, Boron M. Palivizumab Outcomes Registry Group. Prevention of hospitalization due to respiratory syncytial virus: results from the Palivizumab Outcomes Registry. J Perinatol 2008; 28: 511-7.

[40] Feltes TF, Cabalka AK, Meissner HC, et al. Cardiac Synagis Study Group. Palivizumab prophylaxis reduces hospitalization due to respiratory syncytial virus in young children with 
hemodynamically significant congenital heart disease. J Pediatr 2003; 143: 532-40.

[41] Cohen SA, Zanni R, Cohen A, Harrington M, VanVeldhuisen P, Boron ML. Palivizumab Outcomes Registry Group. Palivizumab use in subjects with congenital heart disease: results from the 20002004 Palivizumab Outcomes Registry. Pediatr Cardiol 2008; 29: 382-7.

Received: March 07, 2014

Revised: May 27, 2014

Accepted: May 28, 2014

(c) Bernhard Resch; Licensee Bentham Open.

This is an open access article licensed under the terms of the Creative Commons Attribution Non-Commercial License (http://creativecommons.org/licenses/by-nc/3.0/) which permits unrestricted, non-commercial use, distribution and reproduction in any medium, provided the work is properly cited. 